\title{
A Nucleosome Interaction Module Is Required for Normal Function of Arabidopsis thaliana BRAHMA
}

\author{
S. Farrona, L. Hurtado and J. C. Reyes*
}

\author{
Centro Andaluz de Biología \\ Molecular y Medicina \\ Regenerativa and Instituto \\ de Bioquímica Vegetal y \\ Fotosintesis, Consejo Superior \\ de Investigaciones Científicas \\ Américo Vespucio s/n \\ E-41092 Sevilla, Spain
}

\begin{abstract}
The BRAHMA (BRM) gene encodes the SNF2-type ATPase of the putative Arabidopsis thaliana SWI/SNF chromatin remodelling complex. This family of ATPases is characterized by the presence of a conserved catalytic domain and an arrangement of auxiliary domains, whose functions in the remodelling activity remains unclear. Here, we characterize, at the molecular and functional level, the carboxy-terminal part of Arabidopsis BRM. We have found three DNA-binding regions that bind various free DNA and nucleosomal probes with different specificity. One of these regions contains an AT-hook motif. The carboxy terminus also contains a bromodomain able to bind histones $\mathrm{H} 3$ and $\mathrm{H} 4$. We propose that this array of domains constitute a nucleosome interaction module that helps BRM to interact with its substrate. We also characterize an Arabidopsis mutant that expresses a BRM protein lacking the last 454 amino acid residues (BRM- $\Delta$ C), encompassing the bromodomain and two of the three DNA-binding activities identified. This mutant displays an intermediate phenotype between those of the wild-type and a null allele mutant, suggesting that the nucleosome interaction module is required for the normal function of BRM but it is not essential for the remodelling activity of BRM-containing SWI/SNF complexes.
\end{abstract}

(C) 2007 Elsevier Ltd. All rights reserved.

Keywords: chromatin; bromodomain; swi/snf complex; AT-hook; seed development

\section{Introduction}

Activation and repression of transcription in eukaryotes require the action of multisubunit complexes able to remodel the structure of the chromatin. ${ }^{1}$ Two groups of chromatin remodelling enzymes have been defined: enzymes that produce post-translational covalent modifications of the histones and enzymes that modify interactions between histones and DNA with the concomitant hydrolysis of ATP. ${ }^{2,3}$ An increasing number of chromatin remodelling complexes harboring one or both activities have been identified and characterized in yeast, Drosophila and humans. The catalytic core of an ATP-dependent chromatin remodelling machine

Present address: S. Farrona, Max Planck Institute for Plant Breeding Research, D-50829 Cologne, Germany.

Abbreviations used: GST, glutathione-S-transferase; EMSA, electrophoretic mobility-shift assay; $4 \mathrm{WJ}$, four-way junction; WT, wild-type.

E-mail address of the corresponding author: jose.reyes@cabimer.es is a DNA-dependent ATPase of the SNF2 family. ${ }^{4,5}$ These proteins have the seven motifs characteristic of DNA and RNA helicases of the SF2 superfamily. In addition, most of the SNF2-like proteins have additional characteristic domains (auxiliary domains) with unclear roles in the remodelling activity. In vitro, these ATP-dependent chromatin remodelling machines are able to increase the accessibility of nucleases to the nucleosomal DNA, move nucleosomes along a DNA template in cis (sliding), transfer a histone tetramer or octamer from one DNA template to another in trans, or create dinucleosome structures from mononucleosomes. However, how ATPases recognize their nucleosomal substrates and how they couple ATP hydrolysis to disruption of histone-DNA interactions is unclear. ${ }^{5,6}$

The first ATP-dependent chromatin remodelling enzyme discovered was the SWI/SNF complex, a multisubunit machine composed of about ten proteins in yeast and metazoans. ${ }^{4}$ Compelling evidence suggests that an SWI/SNF-like complex exists also in plants, although it has not been purified. ${ }^{7-9}$ The ATPases of the SWI/SNF complexes belong to the SWI2/SNF2 subfamily and are characterized by the 
presence of several auxiliary domains: domains I and II (defined for the Drosophila Brahma protein), probably involved in protein-protein interaction, an AT-hook motif and a bromodomain. The AT-hook is a short DNA-binding motif, identified originally in the high-mobility group chromosomal proteins HMGA1a (formerly HMG-I(Y)), that consists of a GRP tripeptide surrounded by basic amino acid residues. ${ }^{10}$ AT-hooks bind the minor groove of DNA with a preference for A+T-rich sequences. The AThook motif of the human SWI/SNF ATPase Brahma (hBRM) has been shown to be required for its function. However, its role in chromatin binding and remodeling activity is unclear. ${ }^{11}$ The bromodomain is a 110 amino acid residue domain present in several families of chromatin proteins, including histone acetyltransferases and ATPases of the SWI2/SNF2 subfamily. The bromodomain is able to recognize specific acetylated lysine residues in histone tails, ${ }^{12}$ although recognition of non-acetylated histones has been reported. ${ }^{13}$

Among the 40 ATPases of the SNF2 family found in Arabidopsis, only four belong to the SWI2/SNF2 subfamily on the basis of phylogenetic analysis of the SNF2 ATPase catalytic domains: SPLAYED, BRAHMA, CHR12, and CHR23. Only one, BRAHMA (BRM, also called AtBRM), presents all the domains that are characteristic of ATPases of SWI/SNF complexes. ${ }^{7,9}$ Arabidopsis brm mutants display a pleiotropic phenotype characterized by slow growing, curled leaves, root growth defects, flower homeotic transformation and sterility. ${ }^{7,9,14}$ Here, we show that the Arabidopsis BRAHMA carboxy-terminal region contains a nucleosome interaction module able to interact with DNA and histones. An Arabidopsis mutant that expresses a truncated protein lacking part of this carboxy-terminal region behaves as a hypomorphic allele, suggesting that this region is not indispensable for the BRM remodelling activity but it is required for the normal function of the protein.

\section{Results}

\section{Arabidopsis BRM presents several nucleosomal binding activities}

As previously indicated, the AT-hook motif consists of a GRP tripeptide surrounded by basic amino acid residues. ${ }^{10}$ Arabidopsis BRM contains a consensus AT-hook motif between residues 1606 and 1618 with the sequence RKRGRPKSKK. We decided to test whether a fragment of BRM harbouring this motif was able to bind DNA. To address this issue, we constructed a plasmid-bearing DNA encoding amino acid residues 1568-1919 of Arabidopsis BRM fused to glutathione-S-transferase (GST-ATh1) (Figure 1(a)). The recombinant protein was expressed in Escherichia coli, purified and used for electrophoretic mobility-shift assays (EMSA). GSTATh1 was able to bind different DNA probes with similar affinity, including an A+T-rich DNA frag- (a)

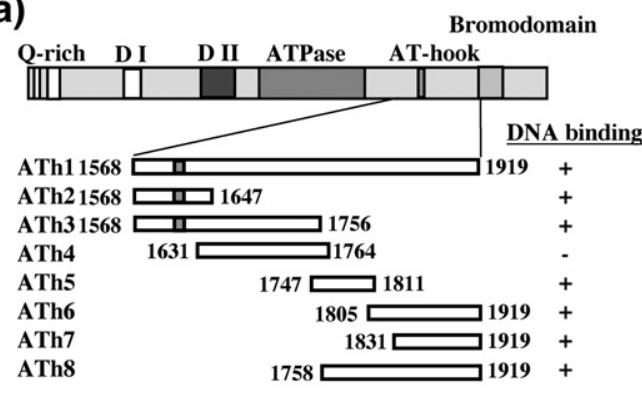

(c)

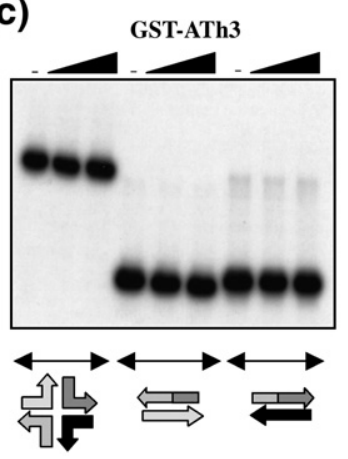

(b)
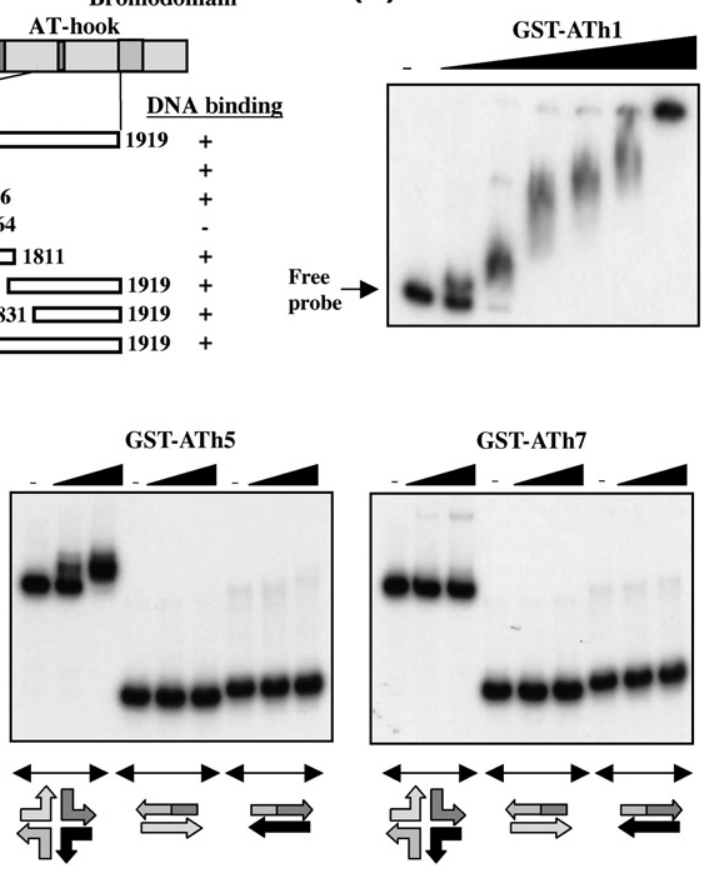

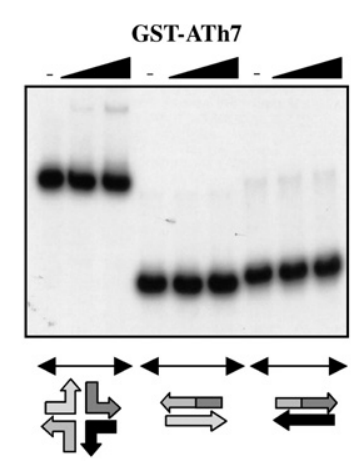

Figure 1. Three different DNAbinding regions are present in BRM. (a) A representation of the domains structure of BRM. Fragments of BRM tested for DNA binding activity by EMSA were designated ATh1 to ATh8. Numbers correspond to amino acid positions. The DNA binding activity of all the GST-ATh recombinant proteins was tested as indicated in (b). Binding activity is denoted by $(+)$, while absence of binding is indicated by $(-)$. Q-rich, glutamine-rich region; DI, domain I; DII, domain II; ATPase, DNAdependent ATPase domain; AThook, DNA-binding domain. (b) GST-ATh1 protein binds DNA. Binding reactions contained a ${ }^{32} \mathrm{P}$ labelled $305 \mathrm{bp}$ DNA fragment of the Arabidopsis plastocyanin S/MAR and increasing quantities (1-37.5 pmol) of recombinant GSTATh1 protein. The resulting complexes were resolved by native $6 \%$ $(\mathrm{w} / \mathrm{v})$ polyacrylamide gel electrophoresis. Gels were dried, and subjected to autoradiography. (c) Interaction of GST-ATh3, GST-ATh5, GST-ATh7 with four-way-junction (4WJ) DNA. Binding reactions contained a radiolabelled 4WJ DNA, ${ }^{16} 100$-fold excess of poly [(dI-C)] competitor DNA and $10 \mathrm{pmol}$ or $20 \mathrm{pmol}$ of the indicated recombinant protein. The resulting complexes were resolved by native $6 \%$ polyacrylamide gel electrophoresis. The gels were dried, and subjected to autoradiography. Representations of the structure of the probes used are shown below the gel images. Control linear probes contain the same sequence as the four arms of the $4 \mathrm{WJ}$ probe, in order to verify that binding is sequence-independent. 
ment of the scaffold/matrix attachment region (S/MAR) of the Arabidopsis plastocyanin locus, ${ }^{15}$ or a non-A + T-rich sequence of the coding region of a bacterial gene (Figure 1(b) and data not shown). The GST-ATh1 protein did not retard ssDNA or RNA probes significantly (data not shown). To further map the minimal region of interaction with the DNA, GST fusion proteins containing different deletions of the ATh1 fragment were expressed in E. coli and purified. DNA-binding activity of these polypeptides was determined by EMSA (Figure 1(a)). According to the presence of a consensus AThook motif, the GST-ATh2 and GST-ATh3 proteins were able to bind DNA. Furthermore, GST-ATh3 DNA binding activity was more efficiently competed by poly $[\mathrm{d}(\mathrm{A}-\mathrm{T})]$ than by poly $[(\mathrm{dC}-\mathrm{G})]$, as expected for the activity of the AT-hook motif (data not shown). Unexpectedly, GST-ATh5 to GST-ATh8 fusion proteins, which lack a consensus AT-hook, were also able to bind DNA with efficiency comparable to that of the GST-ATh1 protein (Supplementary Data Figure 1). GST, or GST-ATh4 were not able to bind DNA. Therefore, our results indicate that other regions of BRM, in addition to the consensus AT-hook motif, are able to interact with DNA.

A common feature of HMGA proteins is the ability to interact with a four-way junction (4WJ) DNA, ${ }^{10}$ a synthetic, cruciform-like structure that is considered a model for distorted DNA. ${ }^{16}$ Therefore, we tested the ability of GST-ATh3, GST-ATh5 and GST-ATh7 to interact with 4WJ DNA by EMSA. As shown in Figure 1(c), only the GST-ATh5 protein was able to interact specifically with 4 WJ DNA but not with double-stranded linear DNA of the same sequence. These assays were carried out in the presence of 100-fold excess of cold competitor DNA (poly $[\mathrm{d}(\mathrm{I}-\mathrm{C})]$ in order to demonstrate higher specificity for $4 \mathrm{WJ}$ than for linear DNA of the same sequence. Therefore, in this assay none of the proteins were able to retard the double stranded oligonucleotides.

Chromatin is the natural substrate of BRM. Therefore, we tested whether the different fragments of BRM that bind free DNA were able to interact with nucleosomal DNA. For that, mononucleosomes were reconstituted using HeLa cell histone octamers and DNA probes of $195 \mathrm{bp}, 168 \mathrm{bp}$ and $146 \mathrm{bp}$ of the $5 \mathrm{~S}$ rRNA gene of Lytechinus variegates. These DNA templates can position a nucleosome rotationally and translationally after in vitro reconstitution. ${ }^{17-19}$ The $195 \mathrm{bp}$ nucleosome contains free linker DNA at the edge of the nucleosome and $168 \mathrm{bp}$ is the length of DNA protected from nucleases digestion by the histone octamer plus the histone H1 (chromatosome). Therefore, histone $\mathrm{H} 1$ is able to bind this nucleosomal substrate. Finally, $146 \mathrm{bp}$ is the length of DNA protected by the histone octamer and, thus, this nucleosomal substrate lacks linker DNA. Reconstituted nucleosomes were subjected to electrophoresis in agarose gels in the presence of increasing amounts of different GST fusion proteins. Interestingly, while GST-ATh3, and GST-ATh5 bound the 195 bp nucleosomes, they were unable to bind the $168 \mathrm{bp}$ or the $146 \mathrm{bp}$ nucleosomes (Figure 2). Irrespectively of the amount of protein used, a unique shifted complex was observed, suggesting the presence of a single binding site in the $195 \mathrm{bp}$ nucleosomal probe (Figure 2 and data not shown). The GST-ATh7 protein bound the three nucleosomal substrates. In contrast to GST-ATh3 and GST-ATh5, GST-ATh7 provoked a diffuse smeared binding, suggesting the presence of several binding sites in the probes. In addition, a smeared binding signal may indicate instability of the protein-nucleosome complex during electrophoresis. Taken together, our data indicate that the region of BRM encompassing residues 1568-1919 contains at least three DNAbinding activities with different characteristics.

\section{The bromodomain of Arabidopsis BRM binds histones}

The bromodomain of Arabidopsis BRM and its Oryza sativa and Zea mays counterparts are significantly divergent with respect to bromodomains of other metazoan or fungal proteins, or even when compared to bromodomains of other plant proteins. In fact, most of the residues involved in the interaction with acetylated lysine residues in the human P/CAF (V752, A757, Y760, Y802, N803 and Y809) or

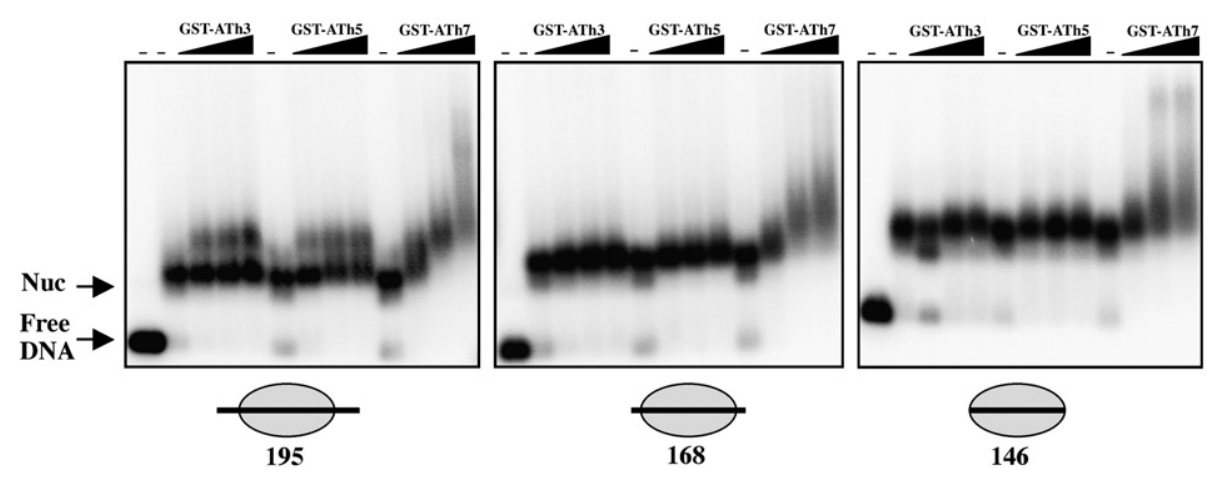

Figure 2. BRM binds nucleosomal DNA. Interaction of GST-ATh3, GST-ATh5, GST-ATh7 (see Figure 1(a)) with nucleosomes. Binding reactions contained mononucleosomes assembled on radiolabeled $195 \mathrm{bp}, 168 \mathrm{bp}$ or $146 \mathrm{bp}$ DNA fragments and increasing quantities of the indicated recombinant proteins. The resulting complexes were resolved by electrophoresis on $1 \%(\mathrm{w} / \mathrm{v})$ agarose gels, transferred to nitrocellulose filters and visualized by autoradiography. 
in the S. cerevisiae GCN5 (P351, F352 V356, V361, Y364, N407, Y413) proteins s $^{20,21}$ are not conserved in Arabidopsis BRM (Figure 3). In order to investigate whether the Arabidopsis BRM bromodomain is able to bind histones, we performed a far-Western-type overlay assay. Purified calf thymus histones were resolved by SDS-PAGE, transferred to a nitrocellulose membrane and incubated with either GST or the GST-BRM $1876-2060$ fusion protein produced in E. coli. GST-BRM 1876-2060, but not GST alone, bound strongly to histones $\mathrm{H} 3$ and H4 (Figure 4). These results demonstrate that the BRM bromodomain binds histones. However, we have not been able to detect preferential binding of this domain to acetylated forms of $\mathrm{H} 3$ or $\mathrm{H} 4$ (data not shown).

\section{Deletion of the carboxy-terminal part of BRM partially abolishes its function}

Earlier, we reported the phenotype of brm null mutant plants. ${ }^{7}$ In order to investigate the role of the carboxy-terminal domains of BRM, we looked for T-DNA insertions that mapped in the $3^{\prime}$ region of the gene. Line SALK_088462 contained a T-DNA insertion in the 11th intron and this mutant allele was named brm-3 (Figure 5(a)). RT-PCR experiments using oligonucleotides 1 and 2 corresponding to a region upstream of the T-DNA insertion amplified normal amounts of BRM mRNA (Figure 5(b)). However, a transcript was not detectable using oligonucleotides 3 and 4, which hybridize downstream of the T-DNA insertion point. These data suggest that a truncated BRM mRNA is transcribed in the homozygous brm-3 plants. We tested by immunoblot

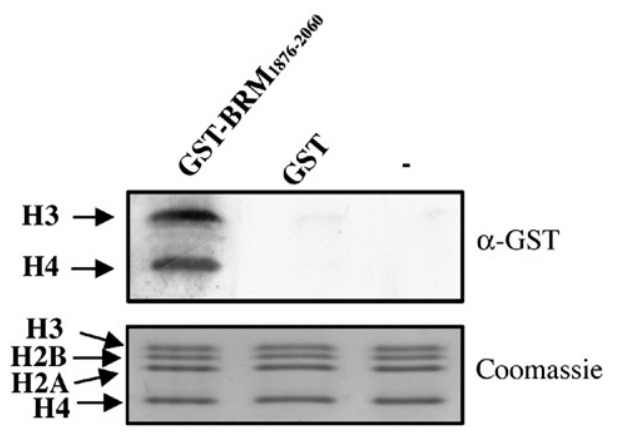

Figure 4. BRM bromodomain interacts with histones $\mathrm{H} 3$ and H4. Total histones were resolved by SDS-PAGE and either stained with Coomassie brilliant blue or transferred to nitrocellulose filters. The membrane was cut to separate the lanes, and each lane was incubated with either GST-BRM $1876-2060$, GST or without added protein. After washing, retained proteins were detected using anti-GST antibodies and secondary anti-rabbit antibodies using standard Western blotting procedures.

whether a truncated protein was present in these plants. For that, two different antibodies, one against the C-terminal part of BRM $(\alpha-B R M-C)$ and another against the N-terminal region $(\alpha-B R M-N)$ were used. As shown in Figure 5(c), both antibodies recognized a polypeptide of about $250 \mathrm{kDa}$ in nuclear extracts from wild-type (WT) plants. However, a polypeptide of approximately $200 \mathrm{kDa}$ was detected in extracts of homozygous brm-3 plants using the $\alpha$-BRM-N antibody, but not using the $\alpha$-BRM-C antibody. Furthermore, similar amounts of both

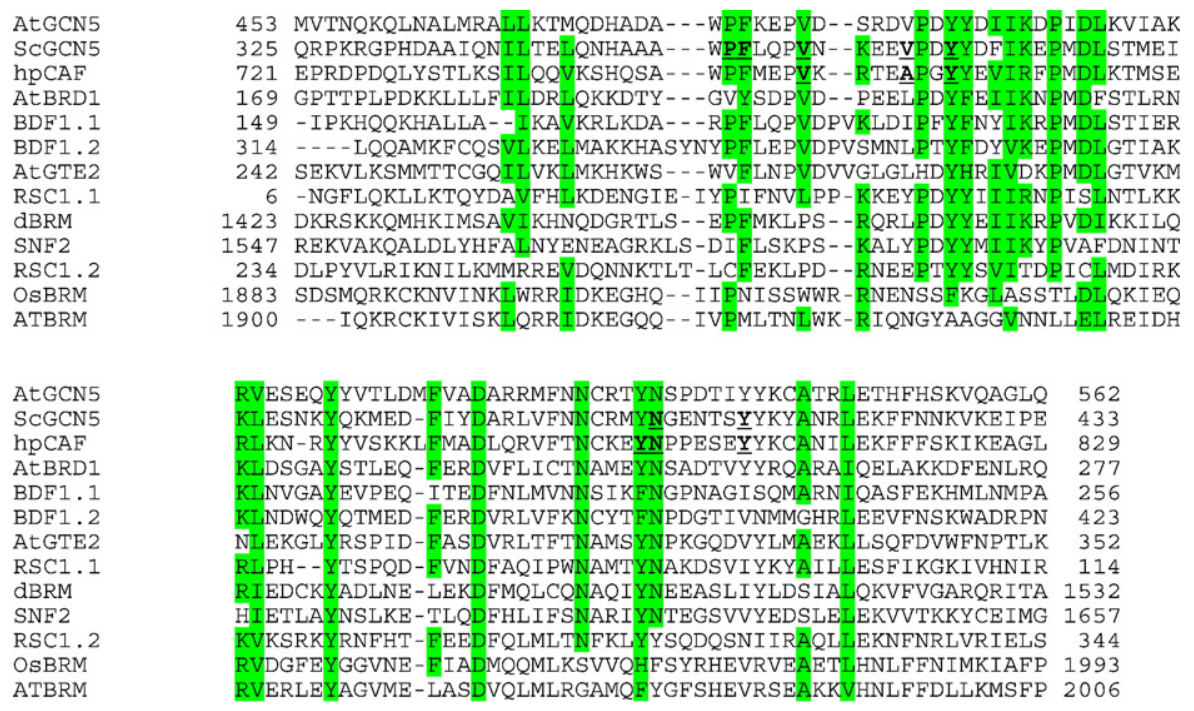

Figure 3. Amino acid sequence alignment of bromodomains from different origins. Residues identical or conserved in at least nine of the 13 sequences are highlighted in green. Conservative changes were defined as those that have a value higher than +2 in the BLOSUM62 scoring matrix (i.e. E-D, R-K, L-I, V-I, Y-F, Y-H, Y-W). Residues of pCAF and ScGCN5 involved in the interaction with acetylated lysine are highlighted in bold-face and underlined. OsBRM, Oryza sativa BRM, (LOC_Os02g02290, XP_463856); AtGCN5, Arabidopsis thaliana GCN5 (AT3G54610); AtBRD1, A. thaliana BRD1 (AT1G20670); AtGTE2, A. thaliana AtGTE2 (AT5G10550); dBRM, Drosophila BRM (P25439); SNF2, S. cerevisiae SNF2 (P22082); ScGCN5, S. cerevisiae GCN5 (Q03330); hpCAF, human pCAF (NP_003875); RSC1.1, first bromodomain of S. cerevisiae RSC1; RSC1.2, second bromodomain of S. cerevisiae RSC1 (NP_011570); BDF1.1, first bromodomain of S. cerevisiae BDF1; BDF1.2, second bromodomain of S. cerevisiae BDF1 (P35817); AtBRM, A. thaliana BRM (CAG28313). 
(a)

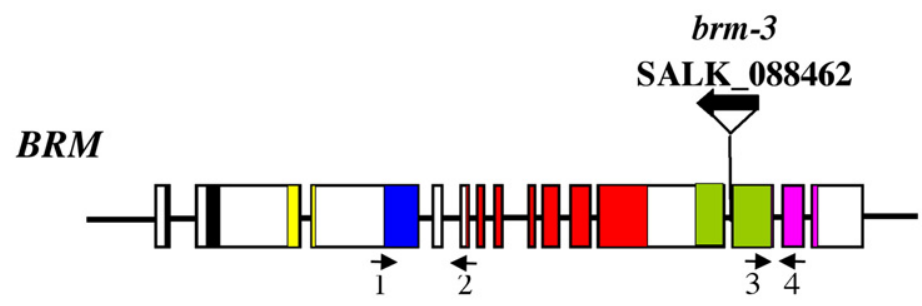

(b)

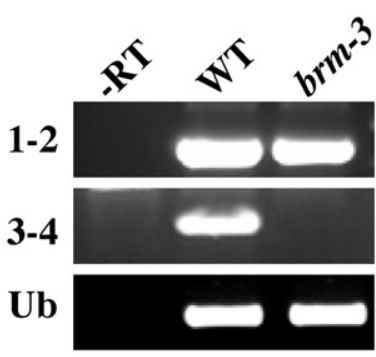

(c)

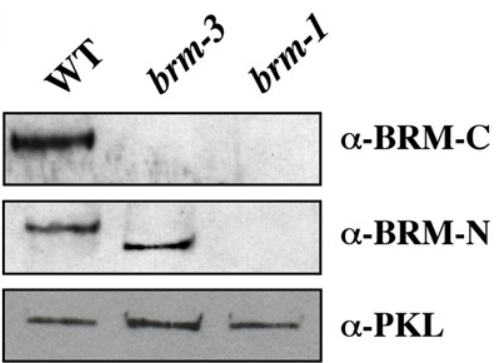

(d)

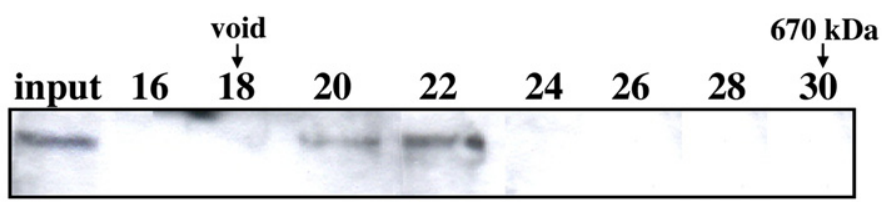

(e)

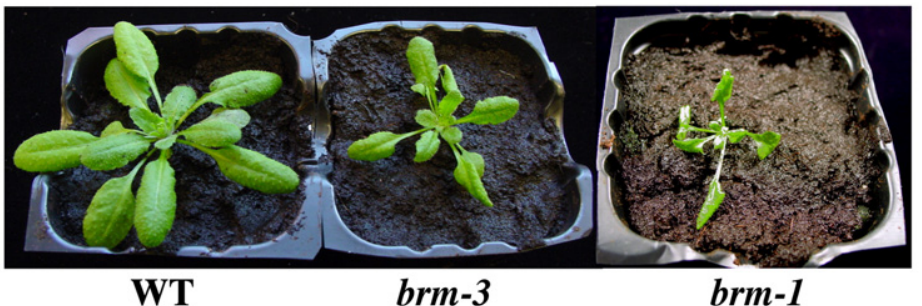

Figure 5. Molecular characterization of brm-3 mutant allele. (a) Molecular structure of the BRM locus and site of T-DNA integration. Colours represent the location of DNA regions encoding the different protein domains of BRM. Black, glutamine-rich region; yellow, domain I; blue, domain II; red, ATPase domain; green, DNA-binding domains; pink, bromodomain. Oligonucleotides used for RT-PCR analysis are numbered and indicated by arrows. (b) RT-PCR analysis of the amount of BRM transcript found in wild-type (WT) and brm-3 plants. PCR reactions were carried out with the indicated oligonucleotide pairs. Reactions without retrotranscriptase were carried out as control (-RT). Amplification of the Ubiquitin (Ub) mRNA was used as a control. (c) Immunoblot analysis of the level of BRM protein. Nuclear extracts from WT, brm-1 and brm-3 inflorescences were subjected to immunoblotting using antibodies against the C-terminal ( $\alpha$ BRM-C) or the $\mathrm{N}$-terminal $(\alpha \mathrm{BRM}-\mathrm{N})$ parts of BRM. Antibodies against the nuclear protein PICKLE were used as a control ( $\alpha$-PKL). (d) Gel-filtration analysis of nuclear extracts of brm-3 seedlings. Fractions from chromatography through a Superose 6 HR column were separated by

SDS-PAGE and examined by Western blotting using $\alpha$ BRM-N serum. Fraction numbers are given across the top and arrows indicate elution position of molecular mass standards. (e) Whole 25 days old WT (Columbia), brm-3, and brm-1 plants grown under long day conditions.

polypeptides were detected by the $\alpha$-BRM-N antibody in extracts of heterozygous brm-3 plants (data not shown). Our data indicate that the brm-3 allele expresses a truncated BRM polypeptide lacking the last 454 residues, including the bromodomain and the DNA-binding domains contained in the ATh5 and ATh7 fragments. We have named this protein $\mathrm{BRM}-\Delta \mathrm{C}$. Cell fractionation experiments demonstrated that the BRM- $\Delta \mathrm{C}$ protein maintains its nuclear localization (data not shown). Furthermore, the brm-3 mutation does not interfere with the assembly of the putative BRM complex, as shown by the high apparent native molecular mass (about $1 \mathrm{MDa})$ of the BRM- $\Delta \mathrm{C}$ protein upon gel-filtration chromatography (Figure 5(d)).

We reasoned that comparison of the phenotypic characteristics of null mutants with those of brm-3 plants would provide information about the function of the deleted domains in BRM- $\Delta$ C. brm-3 plants displayed an intermediate phenotype between brm-1 and brm-2 null alleles and WT plants (Figures 5(e) and 6). Thus, homozygous brm-3 plants presented small and slightly epinastic leaves and short roots (Figure 6(b) and (g)). Similar but much more dramatic alterations were found in brm null mutants. ${ }^{7}$ In contrast to brm null plants, under long day conditions, brm-3 flowers did not display any significant abnormality (Figure 6(f)). However, under short day photoperiod, several phenotypes described previously in brm null flowers were observed, such as short petals, a reduction in the number of stamens $(6.0 \pm 0$ in the WT plants versus $4.5 \pm 1.1$ in brm-3 plants) and fused stamens (in $20 \%$ of the flowers) (Figure 6(c) and (d)). About $5 \%$ of the brm-3 flowers also presented unfussed carpels (Figure 6(e)). In contrast to brm null plants, brm-3 homozygous were not sterile but siliques were shorter than those of the WT (Figure 6(h)) and the number of seeds per silique was reduced compared to WT plants $(19 \pm 3(n=15)$ in brm-3 versus $56 \pm 9$ $(n=15)$ in WT plants). We observed that brm-3 seeds floated in water longer that WT seeds, suggesting a hydration problem. When Arabidopsis seeds come into contact with water, there is immediate extrusion of pectinaceous mucilage from the epidermal cells that facilitates seed hydration. We have used Ruthenium red to stain the mucilage capsule surrounding hydrated WT and brm-3 seeds. While all WT seeds 


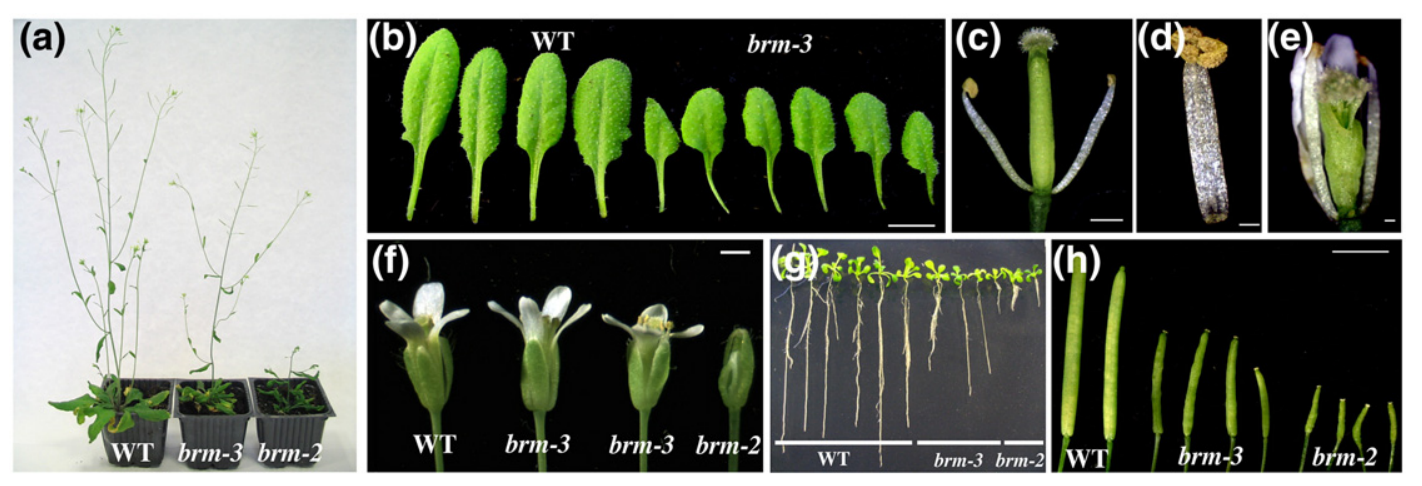

Figure 6. Phenotypic characterization of brm-3 mutants. Vegetative and reproductive phenotypes of brm-3 mutants compared with brm-2 null mutants and WT (Col) plants. ((a), (b), (f)-(h)) Plants were grown under long day conditions (16 h light/8 h dark). ((c)-(e)) Plants were grown under short day conditions (10 h light/14 h dark). Representative pictures are shown. (a) A 40 days old adult WT, brm-3 and brm-2 plants grown in soil. (b) Rosette leaves of brm-3 plants. The scale bar represents $1 \mathrm{~cm}$. (c) Flower with two stamens in a brm-3 plant. Outer organs were removed to make the picture. The scale bar represents $0.5 \mathrm{~mm}$. (d) Fused stamen filaments in brm-3 flowers. The scale bar represents $100 \mu \mathrm{m}$. (e) Short petals and ectopic growth of internal carpelar tissue in brm-3 flowers. The scale bar represents $100 \mu \mathrm{m}$. (f) Side-view of WT, brm-3 and brm-2 flowers. The scale bar represents $1 \mathrm{~mm}$. (g) Roots of WT, brm-3 and brm-2 seedlings grown on a vertical plate for 10 days. (h) Fully expanded WT, brm-2 and brm-3 siliques. The scale bar represents $1 \mathrm{~cm}$.

presented a strongly pink capsule of mucilage (Figure $7(\mathrm{a})$ ), about $60 \%$ of the $\mathrm{brm}-3$ seeds lacked or presented a reduced pink capsule (Figure $7(\mathrm{~b})$ and (c)), suggesting that mucilage synthesis or extrusion is impaired in this mutant. Then, we examined the structure of the seed surface using scanning electron microscopy of whole seeds. As shown in Figure 7(e) and (g), WT seed coat epidermal cells are hexagonal with thickened radial cell walls and a central, volcano-shaped structure known as columella. In most of the brm-3 seeds, this regular structure was disorganised in all or part of the seed surface (Figure 7(f) and (h)). This phenotype has not been observed in brm null mutants because of the sterility of these plants. To corroborate that the seed phenotype was a consequence of a mutation of the BRM gene, we analyzed the seed surface and the mucilage capsule production in transgenic plants where levels of BRM were downregulated by RNAi. ${ }^{9}$ Figure $7(\mathrm{~d})$ and (i) demonstrate that seeds from BRM silenced plants presented the same alterations as $b r m-3$ seeds.

\section{brm-3 mutation has moderate effects on gene expression}

We examined whether deletion of the bromodomain and two DNA-binding domains of BRM affected gene expression. For that, we analyzed the expression level of several genes that have been reported to be deregulated in brm null mutants. Earlier, we showed that the flower homeotic genes PISTILLATA (PI), APETALA2 (AP2), APETALA3 (AP3) and NAC-LIKE, ACTIVATED BY AP3/PI $(N A P)$ are two- to fivefold downregulated in $\mathrm{brm}$ null mutant plants. All these genes were also found to be downregulated in brm-3 homozygous plants, although to a lesser extent (Figure 8). Other genes involved in flower development whose expression is not altered in brm null mutants, such as
APETALA1 (AP1), SEPALLATA3 (SEP3), or AGAMOUS (AG), were also not affected in the brm-3 plants. This result suggests that the intermediate phenotype between the WT and the brm null mutants displayed by the brm-3 plants is consequence of a moderated alteration of the expression of the BRM target genes.

\section{Discussion}

\section{The C-terminal region of BRM contains a nucleosome interaction module}

One of the hallmarks of the ATPases of SWI/SNF complexes is the existence of an AT-hook motif in the C-terminal region, close to the bromodomain. Bourachot et al. demonstrated that in the human BRAHMA protein (hBRM) this motif binds linear DNA. ${ }^{11}$ They showed also that deletion of 58 amino acid residues $\mathrm{N}$-terminal to the bromodomain, containing the AT-hook motif and additional basic amino acid sequences, has no effect on nuclear localization of hBRM but strongly decreases its affinity for the chromatin fraction. We have found that a region of 300 residues upstream of the Arabidopsis BRM bromodomain contains three different DNA-binding activities. The ATh3 region (Figure 1), which contains the AT-hook motive, is able to bind linear DNA probes and nucleosomal templates that contain linker DNA, suggesting that this fragment binds linker DNA at the nucleosomal edge. The ATh5 region is able to bind 4WJ DNA with higher specificity than linear DNA. 4WJ DNA has been proposed to imitate the structure of DNA found at, or near, the entrance and exit points of the nucleosome. As the ATh3 fragment, ATh5 is able to bind to $195 \mathrm{bp}$ templates but not to $168 \mathrm{bp}$ or $146 \mathrm{bp}$ templates. The ATh7 region binds free DNA as well as all nucleosomal substrates, suggesting that this 


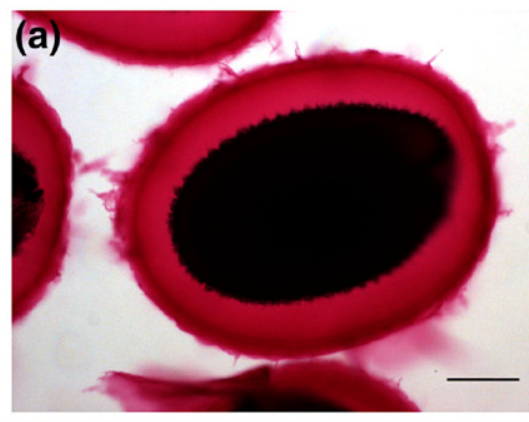

(c)
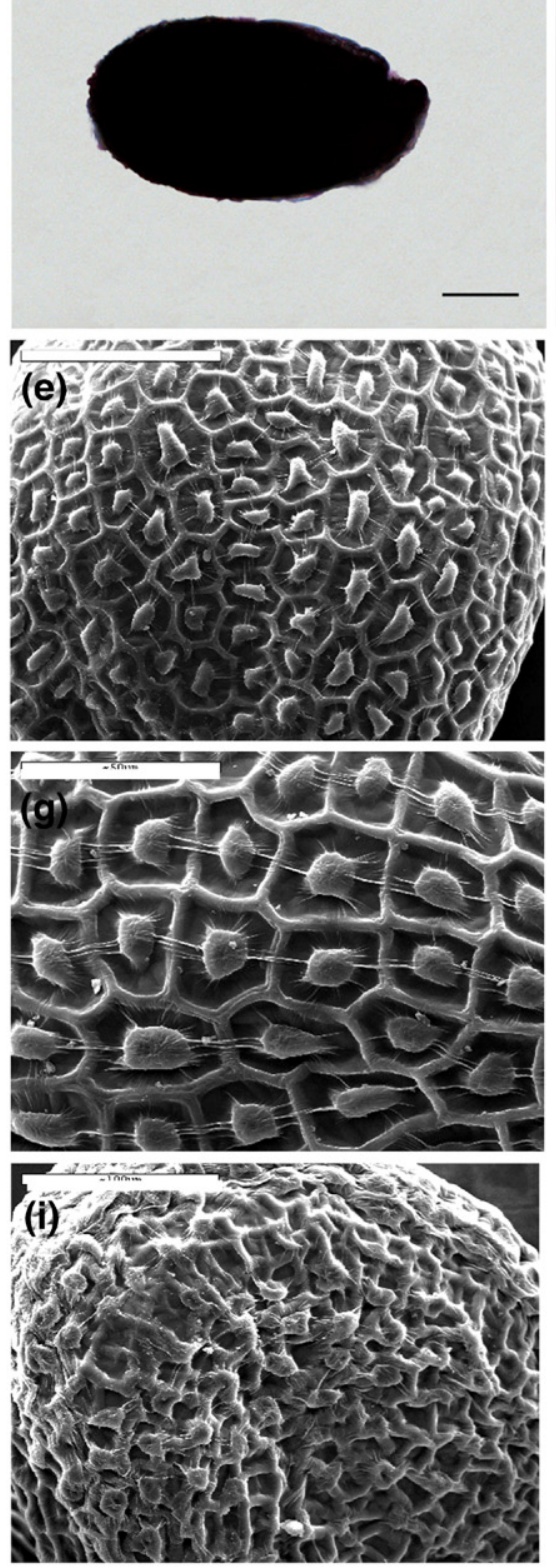

(b)

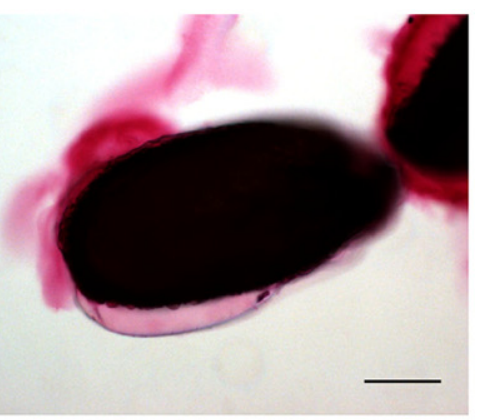

(d)
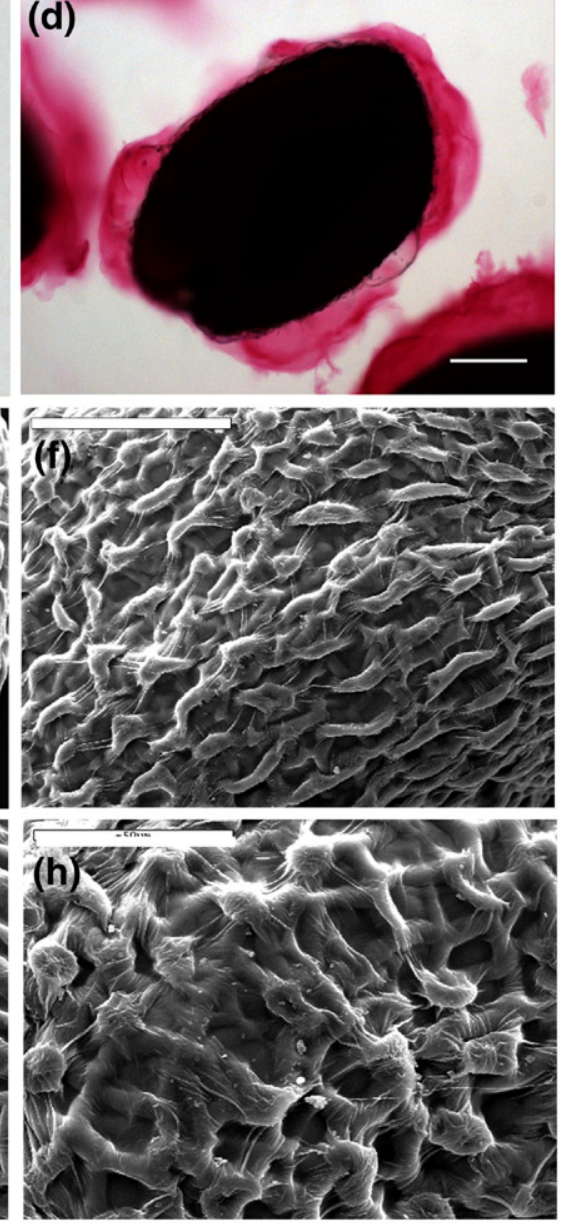

polypeptide interacts with distorted DNA around the histone octamer. The three DNA-binding regions identified are located N-terminally with respect to the BRM bromodomain, which binds histones H3 and H4 (Figure 4). We propose that this array of DNA-binding domains, together with a histonebinding domain, constitutes a substrate (nucleo-
Figure 7. Characterization of WT and brm-3 mutant seeds. (a) Ruthenium red staining of WT, (b) and (c) brm-3 and (d) brm-silenced seeds. Seeds were first shaken in water and then stained as indicated in Materials and Methods. (E-I) Scanning electron micrograph of (e) and (g) WT, (f) and (h) brm-3, and (i) brm-silenced seeds. In (a)-(f), and (i) the scale bars represent $100 \mu \mathrm{m}$. In (g) and (h) the scale bars represent $50 \mu \mathrm{m}$. some) interaction module that allows BRM to interact with the linker DNA, as well as with the nucleosomal DNA and the histone octamer. This nucleosomal interaction module appears to be present in other ATPases of the SWI/SNF complexes from different origins. In the simplest case, the module may be constituted by a single AT-hook fol- 


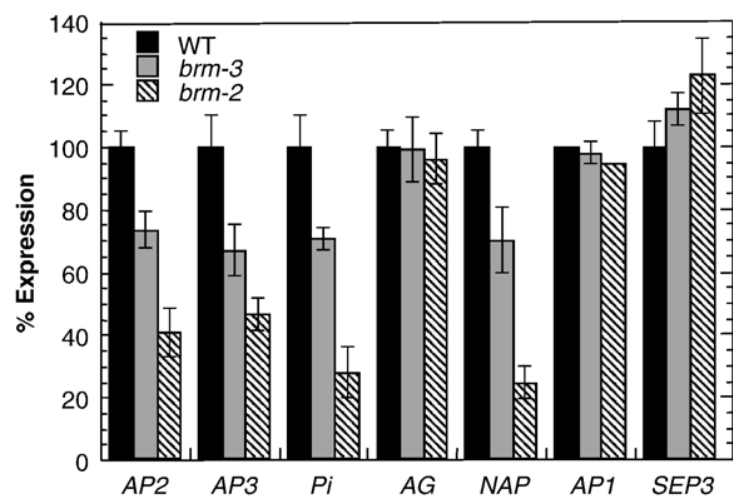

Figure 8. Semi-quantitative RT-PCR analysis of $A P 1$, $A P 2, A P 3, P I, A G, N A P$, and SEP3 transcript levels in WT, brm-3, brm-2 flowers. Total RNA was isolated from inflorescence apices of plants grown under long day conditions. RT-PCR was performed as indicated in Materials and Methods. DNA products were transferred to nylon filters and hybridized with radiolabeled probes for each gene. In order to allow comparison, the expression level of a given gene, was normalized to the GAPC gene transcript level. The level of cDNA amplified in WT plants was considered $100 \%$. Values are the average of three independent experiments. Bars indicate the standard error of the mean.

lowed by a bromodomain (this is the case in hBRM and BRG1 proteins). In the yeast SNF2 protein, two different AT-hook motifs (residues 1445-1457 and 1517-1529) are organized upstream of the bromodomain (1546-1656), although they have not been functionally characterized. The STH1 protein, the ATPase of the yeast RSC complex, does not seem to contain an AT-hook motif. Interestingly, the SMART program $^{22}$ identifies in STH1 a domain distantly related to the DNA-binding domain of the HMGN family of proteins (formerly HMG-14/-17). ${ }^{23}$ This region is only a few amino acid residues upstream of the bromodomain and consequently follows an organization similar to that of the nucleosome interaction module of BRM. A different nucleosome recognition module has been characterized in the $C$ terminal half of the ISWI subfamily of ATPases. ${ }^{24}$ This module is constituted by a SANT domain (involved in $\mathrm{H} 3$ tail binding), a SLIDE domain (involved in DNA binding) and a HAND domain with unknown function. Deletion of the SANT domain partially affects the ISWI remodelling activity in vitro, but deletion of both the SANT and the SLIDE domains abolishes this activity completely. ${ }^{24}$ Therefore, the association of one or more DNA-binding domains with a histone-binding domain seems to be a domain arrangement conserved in several subfamilies of the SNF2 family of ATPases.

\section{Integrity of the nucleosome interaction module is required for normal BRM function}

The brm-3 plants contain a truncated BRM protein lacking the C-terminal 454 amino acid residues (BRM- $\Delta \mathrm{C})$. BRM- $\Delta \mathrm{C}$ lacks the DNA-binding activ- ities of the ATh5 and ATh7 fragments and the bromodomain, but conserves the consensus AT-hook. brm-3 plants display a weak phenotype in comparison to brm-1 and brm-2 null mutants, suggesting that the deleted domains are not essential for BRM remodelling activity but are required for its correct function.

Bromodomain deletion in yeast SWI2/SNF2, or Drosophila Brahma has no obvious phenotype. ${ }^{25-27}$ Consistently, both bromodomain-truncated proteins were assembled correctly in their respective SWI/ SNF complexes. ${ }^{27,28}$ Similarly, we have shown that $\mathrm{BRM}-\Delta \mathrm{C}$ elutes in gel-filtration chromatography associated with a high molecular mass complex, suggesting that the truncated protein can be incorporated into the putative Arabidopsis SWI/SNF complex. This is in agreement with our previous data that indicate that BRM interacts with other members of the plant SWI/SNF complex via the N-terminal region. ${ }^{7,9}$ In vitro experiments showed that yeast SNF2 bromodomain is required for attaching the SWI/SNF complex on acetylated nucleosome arrays. Furthermore, SNF2 bromodomain deletion reduces occupancy of the SWI/SNF complex at the SUC2 promoter in vivo under derepressing conditions, suggesting that the function of the bromodomain is to anchor and to stabilize the SWI/SNF complex to its target promoters. ${ }^{28} \mathrm{SWI} /$ SNF recruiting seems to be carried out by specific transcription factors. ${ }^{29-31}$ However, contribution of the bromodomain to the targeting process, addressing the remodelling complexes to loci with specific acetylation patterns, cannot be excluded. These data suggest that the bromodomain has an accessory role and not an essential role in the remodelling activity. This is consistent with the intermediate phenotype and the moderated effect on gene expression observed in brm-3 plants. Thus, expression of genes strongly deregulated in the absence of BRM is altered only moderately in brm-3 plants. brm-3 mutant plants presented a severe defect in the structure of the seed coat, including disorganization of the epidermal cell layer and impaired extrusion of mucilage. Similar results have been described for mutant plants with ap 2 weak alleles. ${ }^{32}$ Therefore, one obvious possibility is that the moderate downregulation of AP2 observed in brm-3 plants is responsible of this seed alteration.

Taken together, these data suggests that the Arabidopsis SWI/SNF complexes containing the $\mathrm{BRM}-\Delta \mathrm{C}$ protein are able to be recruited to its gene targets and to remodel the chromatin; however, the efficiency or the yield of these processes is probably decreased. How can the BRM complex function partially in the absence of the BRM nucleosome interaction module? One possible explanation is that other BRM complex components may contain nucleosome and histone-binding domains. Thus, mammalian and yeast SWI/SNF-like complexes contain several histone-binding motifs and nonspecific DNA-binding domains. ${ }^{33}$ Interestingly, the BRM paralogous protein SPLAYED also contains an AT-hook motif but lacks the bromodomain. ${ }^{34} \mathrm{brm}$ 
and syd mutants exhibit different phenotypes and we have proposed that the presence of a bromodomain in BRM may address the BRM-containing SWI/SNF complexes to a specific subset of genes different from that controlled by SYD. ${ }^{9}$ However, the results presented here argue against a role of the bromodomain in targeting. Therefore, specificity may be conferred by interaction with specific transcription factors or with different subsets of other SWI/SNF complex subunits. In this sense, we showed earlier that BRM interacts strongly with AtSWI3C and weakly with AtSWI3B, ${ }^{7}$ while Bezhani et al. have reported that SYD interacts with AtSWI3A and AtSWI3B, and only weakly with AtSWI3C. ${ }^{35}$

\section{Materials and Methods}

\section{Plant material and growth conditions}

Wild-type Arabidopsis thaliana and T-DNA mutant (Columbia ecotype) plants were grown either in pots containing a mixture of substrate and vermiculite $3.1(\mathrm{v}: \mathrm{v})$ or aseptically in Petri dishes containing Murashige and Skoog medium supplemented with $1 \%(\mathrm{w} / \mathrm{v})$ sucrose and $0.37 \%(\mathrm{w} / \mathrm{v})$ Phytagel (Sigma). Plants were grown in a cabinet under long day (16 h light/ $8 \mathrm{~h}$ dark) or short day (10 h light/14 h dark) conditions at $23{ }^{\circ} \mathrm{C}$ (day)/20 ${ }^{\circ} \mathrm{C}$ (night), 70\% relative humidity, and a light intensity of $130 \mu \mathrm{E} \mathrm{m}^{-2} \mathrm{~s}^{-1}$ supplied by white fluorescent lamps. Line SALK_088462 from the Salk collection ${ }^{36}$ was obtained from NASC (Nottingham University, UK).

\section{Analysis of T-DNA insertion lines}

The mutant line was backcrossed twice with WT before phenotypic analysis. Plant DNA was extracted from one or two young leaves as described. ${ }^{37}$ Southern blotting experiments demonstrated that the SALK_088462 line contains a single T-DNA insertion. The location of the T-DNA insertion in the mutant line was verified by PCR amplification of genomic DNA with T-DNA left border LBa1 (5'-TGGTTCACGTAGTGGGCCATCG-3') and $B R M$-specific primers. The amplified DNA fragment was then sequenced to identify the T-DNA insertion point within the gene. A second PCR was performed using two gene-specific primers. Only the wild-type allele was amplified by this PCR, whereas no amplification product was detectable from plants homozygous for the insertion. Segregation analysis was carried out by PCR-analysis of the genotype of 30 to 40 offspring obtained after selfpollination of heterozygous plants.

\section{Recombinant protein expression and purification}

Recombinant GST fusion proteins were used for farWestern experiments, and for band-shift assays. For that, PCR-generated DNA fragments were cloned into the appropriate restriction sites of plasmids $\mathrm{PGEX}-4$ T or $\mathrm{pGEX}-6 \mathrm{P}$ in-frame with the GST gene. Details of the constructs and oligonucleotides used are available upon request.

Fusion proteins were expressed in E. coli BL21 cells. From $200 \mathrm{ml}$ to 11 of Luria broth culture was grown to an absorbance at $600 \mathrm{~nm}$ of 0.6 , induced with $1 \mathrm{mM}$ IPTG for $2.5 \mathrm{~h}$, harvested by centrifugation, and resuspended in
5-8 ml of PBS (150 mM NaCl, $16 \mathrm{mM} \mathrm{Na}_{2} \mathrm{HPO}_{4}, 4 \mathrm{mM}$ $\mathrm{NaH}_{2} \mathrm{PO}_{4}$ ), supplemented with $4 \mathrm{mM}$ PMSF and $1 \%(\mathrm{v} / \mathrm{v})$ Triton X-100. Cells were broken by sonication on ice and insoluble debris was pelleted by centrifugation. Extracts were mixed with $0.5-1 \mathrm{ml}$ of Glutathione-Sepharose 4B (Amersham) and incubated for $2 \mathrm{~h}$ at $4{ }^{\circ} \mathrm{C}$ with gentle agitation. The beads were transferred to a column and washed extensively with PBS until no more protein was eluted from the column. GST or GST fusion proteins were eluted with $3 \mathrm{ml}$ of $50 \mathrm{mM}$ Tris- $\mathrm{HCl}(\mathrm{pH} 8.0), 10 \mathrm{mM}$ reduced glutathione, and the eluates were dialysed against the appropriate buffer.

\section{Far-Western experiments}

Since the amino acid sequence of animal and Arabidopsis histones $\mathrm{H} 3$ and $\mathrm{H} 4 \mathrm{~N}$-terminal tails are identical, we have used commercial (Roche) calf thymus histones for far-Western experiments. Acid-extracted core histones from calf thymus $(1 \mu \mathrm{g} / \mathrm{lane})$ were resolved by SDS-PAGE and transferred to nitrocellulose membranes. Separated lanes were incubated overnight with $1 \mu \mathrm{g} / \mathrm{ml}$ of GST-BRM ${ }_{1876-2060}$, or GST in PBS, 1\% Tween-20, 5\% $(\mathrm{w} / \mathrm{v})$ BSA. Control lanes without added recombinant proteins were also processed. Next, membranes were incubated with anti-GST polyclonal antibodies and developed as normal immunoblots.

\section{Cell extracts}

Total cell and nuclear extracts were carried out as described. ${ }^{38,39}$ For Western blotting, isolated nuclei were lysed in NIB $^{39}$ supplemented with $1.5 \%(\mathrm{w} / \mathrm{v})$ SDS. Total protein was determined by a modified Lowry procedure using ovalbumin as a standard. ${ }^{40}$ For size-exclusion chromatography, nuclear proteins were extracted with $0.4 \mathrm{M}\left(\mathrm{NH}_{4}\right)_{2} \mathrm{SO}_{4}$ in NIB. After $30 \mathrm{~min}$ at $4{ }^{\circ} \mathrm{C}$, nuclear debris was pelleted by centrifugation and proteins were precipitated by adding $0.3 \mathrm{~g} / \mathrm{ml}$ of $\left(\mathrm{NH}_{4}\right)_{2} \mathrm{SO}_{4}$. The protein pellet was resuspended in NIB and loaded onto a Superose 6 HR 10/30 column (Amersham) for HPLC.

\section{Seeds staining and scanning electron microscopy}

The seed mucilage layer was stained with Ruthenium red. For that, seeds were agitated in water for $1 \mathrm{~min}$ and then stained for $1 \mathrm{~h}$ in a $0.01 \%(\mathrm{w} / \mathrm{v})$ Ruthenium red. For scanning electron microscopy, dried seeds were coated with gold and observed using a JSM5400 (JEOL) scanning electron microscope.

\section{BRM antibodies and immunoblot analysis}

Two different anti-BRM rabbit polyclonal antibodies were used. The $\alpha$-BRM-C antibody ${ }^{9}$ was raised against amino acid residues 2047-2187 of BRM and therefore recognize the C-terminal part of the protein. The $\alpha$-BRM$\mathrm{N}^{7}$ was raised against amino acid residues 307-424 of BRM and therefore recognize the $\mathrm{N}$-terminal part of the protein. Polyclonal rabbit $\alpha$-PKL antibodies will be described elsewhere. Cell extracts were carried out as. ${ }^{9}$ For Western blot analysis, $10 \mu \mathrm{g}$ of nuclear proteins was fractionated by SDS-PAGE and transferred to nitrocellulose membranes. The membrane was blocked with PBS, $0.5 \%(\mathrm{v} / \mathrm{v})$ Tween $20,5 \%(\mathrm{w} / \mathrm{v})$ fat-free milk power and incubated with the appropriate antisera $(1 / 2000(\mathrm{v} / \mathrm{v})$ 
dilution). Enhanced chemiluminescence (ECL) reagents (Amersham) were used for detection.

\section{Band-shift assays}

Binding of GST fusion proteins to DNA was analysed by electrophoresis mobility-shift assays (EMSA) as described. ${ }^{41}$ DNA probes were end-labelled with ${ }^{32} \mathrm{P}$ labelled deoxynucleosides triphosphate using Sequenase version 2.0 (USB, Cleveland, Ohio). Binding assays were performed in $20 \mu \mathrm{l}$ reaction mixtures containing $2 \mathrm{ng}$ of labelled DNA, $100 \mathrm{mM}$ Tris- $\mathrm{HCl}(\mathrm{pH}$ 7.5), $2 \mathrm{mM} \mathrm{MgCl}$, $1 \mathrm{mM}$ DTT, $35 \mathrm{mM} \mathrm{NaCl}, 10 \%$ (v/v) glycerol, and different amounts of the indicated protein. After incubation at $25^{\circ} \mathrm{C}$ for $15 \mathrm{~min}$, the reaction mixtures were subjected to electrophoresis in non-denaturing $6 \%$ polyacrylamide $/ 10 \%$ glycerol gel in $0.25 \times$ Tris-borate-EDTA at $4{ }^{\circ} \mathrm{C}$. Gels were transferred to Whatman 3MM paper, dried, and subjected to autoradiography.

Binding to $4 \mathrm{WJ}$ and double-stranded oligonucleotides ${ }^{16}$ was done under similar conditions, except that $2 \mu \mathrm{g}$ of poly $[(\mathrm{dI}-\mathrm{C})]$ were included in the reaction mixture. The $4 \mathrm{WJ}$ substrate was prepared as described. ${ }^{42}$

For protein-nucleosome interaction analysis, H1depleted nucleosomes were assembled by octamer transfer as described, ${ }^{43}$ using DNA probes of $195 \mathrm{bp}, 168 \mathrm{bp}$ and $146 \mathrm{bp}$ of the $5 \mathrm{~S}$ rRNA gene from Lytechinus variegatus. Probes of different size were generated by standard PCR techniques and end-labelled with phage T4 polynucleotide kinase and $\left[\gamma_{-}{ }^{32} \mathrm{P}\right]$ ATP $(3000 \mathrm{Ci} / \mathrm{mmol})$. GST-BRM derivatives were incubated with approximately $50 \mathrm{fmol}$ of nucleosomes in $25 \mu \mathrm{l}$ of $50 \mathrm{mM}$ Tris- $\mathrm{HCl}(\mathrm{pH}$ 7.4), $50 \mathrm{mM} \mathrm{NaCl}, 0.5 \mathrm{mM}$ PMSF, $10 \%$ glycerol for $15 \mathrm{~min}$ at room temperature. The samples were then resolved on $1 \%$ agarose gels in $0.5 \times \mathrm{TBE}$ at $4{ }^{\circ} \mathrm{C}$. Then DNA and proteins were transferred to nitrocellulose filters by standard DNA blotting techniques, and subjected to autoradiography.

\section{RT-PCR analysis}

RNA was isolated using the RNeasy Mini Kit (Qiagen). For semi-quantitative RT-PCR, $5 \mu \mathrm{g}$ of total RNA was used to generate the first-strand cDNA with the SuperScript First-Strand Synthesis System for RT-PCR kit (Invitrogen). PCR amplification was performed using $2 \mu \mathrm{l}$ out of $20 \mu \mathrm{l}$ of the RT reaction and specific primers for each analyzed gene. From 15 to 25 amplification PCR cycles were employed and DNA products were detected by Southern blot hybridization. The number of PCR cycles in the different experiments was shown to be in the linear range of the reaction. Primer sequences and details of the probes used for DNA gel blot experiments are available upon request.

\section{Acknowledgements}

We thank the Salk Institute Genomic Analysis Laboratory, and the European Arabidopsis Stock Centre (NASC, Nottingham University, UK) for providing the T-DNA insertion mutant used in this study. We thank José L. Crespo, Marika Lindahl, Javier Florencio, Mario García-Domínguez, Rosana March-Díaz and Yuhai Cui for critical reading of the manuscript. S. F. and L. H. were recipients of fellowships from the Spanish Ministerio de Educación y Ciencia. This work was supported by Ministerio de Educación y Ciencia (grants BMC200203198, BFU2005-01047) and by Junta de Andalucía (group CV1-284).

\section{Supplementary Data}

Supplementary data associated with this article can be found, in the online version, at doi:10.1016/ j.jmb.2007.07.012

\section{References}

1. Mellor, J. (2005). The dynamics of chromatin remodeling at promoters. Mol. Cell, 19, 147-157.

2. Margueron, R., Trojer, P. \& Reinberg, D. (2005). The key to development: interpreting the histone code? Curr. Opin. Genet. Dev. 15, 163-176.

3. Cairns, B. R. (2005). Chromatin remodeling complexes: strength in diversity, precision through specialization. Curr. Opin. Genet. Dev. 15, 185-190.

4. Smith, C. L. \& Peterson, C. L. (2005). ATP-dependent chromatin remodeling. Curr. Top. Dev. Biol. 65, 115-148.

5. Saha, A., Wittmeyer, J. \& Cairns, B. R. (2006). Chromatin remodelling: the industrial revolution of DNA around histones. Nature Rev. Mol. Cell Biol. 7, 437-447.

6. Durr, H., Flaus, A., Owen-Hughes, T. \& Hopfner, K. P. (2006). Snf2 family ATPases and DExx box helicases: differences and unifying concepts from high-resolution crystal structures. Nucl. Acids Res. 34, 4160-4167.

7. Hurtado, L., Farrona, S. \& Reyes, J. C. (2006). The putative SWI/SNF complex subunit BRAHMA activates flower homeotic genes in Arabidopsis thaliana. Plant Mol. Biol. 62, 291-304.

8. Sarnowski, T. J., Rios, G., Jasik, J., Swiezewski, S., Kaczanowski, S., Li, Y. et al. (2005). SWI3 subunits of putative SWI/SNF chromatin-remodeling complexes play distinct roles during Arabidopsis development. Plant Cell, 17, 2454-2472.

9. Farrona, S., Hurtado, L., Bowman, J. L. \& Reyes, J. C. (2004). The Arabidopsis thaliana SNF2 homolog AtBRM controls shoot development and flowering. Development, 131, 4965-4975.

10. Reeves, R. \& Beckerbauer, L. (2001). HMGI/Y proteins: flexible regulators of transcription and chromatin structure. Biochim. Biophys. Acta, 1519, 13-29.

11. Bourachot, B., Yaniv, M. \& Muchardt, C. (1999). The activity of mammalian brm/SNF2alpha is dependent on a high-mobility- group protein I/Y-like DNA binding domain. Mol. Cell Biol. 19, 3931-3939.

12. Zeng, L. \& Zhou, M. M. (2002). Bromodomain: an acetyllysine binding domain. FEBS Letters, 513, 124-128.

13. Matangkasombut, O. \& Buratowski, S. (2003). Different sensitivities of bromodomain factors 1 and 2 to histone $\mathrm{H} 4$ acetylation. Mol. Cell, 11, 353-363.

14. Kwon, C. S., Hibara, K., Pfluger, J., Bezhani, S., Metha, H., Aida, M. et al. (2006). A role for chromatin remodeling in regulation of CUC gene expression in the Arabidopsis cotyledon boundary. Development, 133, 3223-3230.

15. van Drunen, C. M., Oosterling, R. W., Keultjes, G. M., Weisbeek, P. J., van Driel, R. \& Smeekens, S. C. (1997). Analysis of the chromatin domain organisation around the plastocyanin gene reveals an MAR-specific 
sequence element in Arabidopsis thaliana. Nucl. Acids Res. 25, 3904-3911.

16. Bianchi, M. E., Beltrame, M. \& Paonessa, G. (1989). Specific recognition of cruciform DNA by nuclear protein HMG1. Science, 243, 1056-1059.

17. Simpson, R. T. \& Stafford, D. W. (1983). Structural features of a phased nucleosome core particle. Proc. Natl Acad. Sci. USA, 80, 51-55.

18. Simpson, R. T., Thoma, F. \& Brubaker, J. M. (1985) Chromatin reconstituted from tandemly repeated cloned DNA fragments and core histones: a model system for study of higher order structure. Cell, 42, 799-808.

19. Dong, F., Hansen, J. C. \& van Holde, K. E. (1990). DNA and protein determinants of nucleosome positioning on sea urchin $5 \mathrm{~S}$ rRNA gene sequences in vitro. Proc. Natl Acad. Sci. USA, 87, 5724-5728.

20. Dhalluin, C., Carlson, J. E., Zeng, L., He, C., Aggarwal, A. K. \& Zhou, M. M. (1999). Structure and ligand of a histone acetyltransferase bromodomain. Nature, 399, 491-496.

21. Owen, D. J., Ornaghi, P., Yang, J. C., Lowe, N., Evans, P. R., Ballario, P. et al. (2000). The structural basis for the recognition of acetylated histone $\mathrm{H} 4$ by the bromodomain of histone acetyltransferase gen $5 p$. EMBO J. 19, 6141-6149.

22. Letunic, I., Copley, R. R., Schmidt, S., Ciccarelli, F. D., Doerks, T., Schultz, J. et al. (2004). SMART 4.0: towards genomic data integration. Nucl. Acids Res. 32, D142-D144.

23. Bustin, M., Trieschmann, L. \& Postnikov, Y. V. (1995). The HMG-14/-17 chromosomal protein family: architectural elements that enhance transcription from chromatin templates. Semin. Cell Biol. 6, 247-255.

24. Grune, T., Brzeski, J., Eberharter, A., Clapier, C. R., Corona, D. F., Becker, P. B. \& Muller, C. W. (2003). Crystal structure and functional analysis of a nucleosome recognition module of the remodeling factor ISWI. Mol. Cell, 12, 449-460.

25. Laurent, B. C., Treich, I. \& Carlson, M. (1993). The yeast SNF2/SWI2 protein has DNA-stimulated ATPase activity required for transcriptional activation. Genes Dev. 7, 583-591.

26. Laurent, B. C., Treitel, M. A. \& Carlson, M. (1991). Functional interdependence of the yeast SNF2, SNF5 and SNF6 proteins in transcriptional activation. Proc. Natl Acad. Sci. USA, 88, 2687-2691.

27. Elfring, L. K., Daniel, C., Papoulas, O., Deuring, R. Sarte, M., Moseley, S. et al. (1998). Genetic analysis of brahma: the Drosophila homolog of the yeast chromatin remodeling factor SWI2/SNF2. Genetics, 148, 251-265.

28. Hassan, A. H., Prochasson, P., Neely, K. E., Galasinski, S. C., Chandy, M., Carrozza, M. J. \& Workman, J. L. (2002). Function and selectivity of bromodomains in anchoring chromatin-modifying complexes to promoter nucleosomes. Cell, 111, 369-379.

29. Hsiao, P. W., Fryer, C. J., Trotter, K. W., Wang, W. \&
Archer, T. K. (2003). BAF60a mediates critical interactions between nuclear receptors and the BRG1 chromatin-remodeling complex for transactivation. Mol. Cell Biol. 23, 6210-6220.

30. Kadam, S. \& Emerson, B. M. (2003). Transcriptional specificity of human SWI/SNF BRG1 and BRM chromatin remodeling complexes. Mol. Cell, 11, 377-389.

31. Cheng, S. W., Davies, K. P., Yung, E., Beltran, R. J., Yu, J. \& Kalpana, G. V. (1999). c-MYC interacts with INI1/ hSNF5 and requires the SWI/SNF complex for transactivation function. Nature Genet. 22, 102-105.

32. Western, T. L., Burn, J., Tan, W. L., Skinner, D. J., Martin-McCaffrey, L., Moffatt, B. A. \& Haughn, G. W. (2001). Isolation and characterization of mutants defective in seed coat mucilage secretory cell development in Arabidopsis. Plant Physiol. 127, 998-1011.

33. Mohrmann, L. \& Verrijzer, C. P. (2005). Composition and functional specificity of SWI2/SNF2 class chromatin remodeling complexes. Biochim. Biophys. Acta, 1681, 59-73.

34. Wagner, D. \& Meyerowitz, E. M. (2002). SPLAYED, a Novel SWI/SNF ATPase homolog, controls reproductive development in Arabidopsis. Curr. Biol. 12, 85-94.

35. Bezhani, S., Winter, C., Hershman, S., Wagner, J. D., Kennedy, J. F., Kwon, C. S. et al. (2007). Unique, shared, and redundant roles for the Arabidopsis SWI/ SNF chromatin remodeling ATPases BRAHMA and SPLAYED. Plant Cell, 19, 403-416.

36. Alonso, J. M., Stepanova, A. N., Leisse, T. J., Kim, C. J., Chen, H., Shinn, P. et al. (2003). Genome-wide insertional mutagenesis of Arabidopsis thaliana. Science, 301, 653-657.

37. Murray, M. G. \& Thompson, W. F. (1980). Rapid isolation of high molecular weight plant DNA. Nucl. Acids Res. 8, 4321-4325.

38. Martinez-Garcia, J. F., Monte, E. \& Quail, P. H. (1999). A simple, rapid and quantitative method for preparing Arabidopsis protein extracts for immunoblot analysis. Plant J. 20, 251-257.

39. Guilfoyle, T. J. (1995). Isolation and characterization of plant nuclei. Methods Cell Biol. 50, 101-112.

40. Markwell, M. A., Haas, S. M., Bieber, L. L. \& Tolbert, N. E. (1978). A modification of the Lowry procedure to simplify protein determination in membrane and lipoprotein samples. Anal. Biochem. 87, 206-210.

41. Sambrook, J., Fritsch, E. F. \& Maniatis, T. (1989). Molecular Cloning: A Laboratory Manual, 2nd edit. Cold Spring Harbor Laboratory Press, Cold Spring Harbor, NY.

42. Teo, S. H., Grasser, K. D., Hardman, C. H., Broadhurst, R. W., Laue, E. D. \& Thomas, J. O. (1995). Two mutations in the HMG-box with very different structural consequences provide insights into the nature of binding to four-way junction DNA. EMBO J. 14, 3844-3853.

43. Ura, K. \& Wolffe, A. P. (1996). Reconstruction of transcriptionally active and silent chromatin. Methods Enzymol. 274, 257-271. 